\title{
Experimental investigation into material removal mechanisms in High Speed Wire EDM
}

\author{
Kai Oßwald ${ }^{1}$ (1) $\cdot$ Lukas Brandl $^{1} \cdot$ Ingo Lochmahr $^{2}$
}

Received: 29 June 2020 /Revised: 21 September 2020 / Accepted: 15 October 2020 / Published online: 23 October 2020

(C) The Author(s) 2020

\begin{abstract}
High Speed Wire EDM (HSWEDM) is characterized by high relative velocities between its electrodes which appear in almost no other field of electrical discharge machining (EDM). Also, it previously has been described as a hybrid process. Consequently, material removal mechanisms show significant differences compared with other EDM processes. In this research, single discharge craters of HSWEDM processes were examined, and their geometrical features were associated with the underlying parameters. Discharge crater geometries on the workpiece electrode (anode) could be investigated by measurements whilst those on the wire electrode (cathode) were calculated. Results show that the high wire velocity leads to moving foot points on both electrodes. Pulse duration and the type of working medium influence the generation of discharge craters and thus their geometry and the modes of material removal. Static discharges and anodic dissolution could also be identified as material removal mechanisms and characterized in their geometrical properties. However, they are of secondary importance.
\end{abstract}

Keywords Electrical discharge machining $\cdot$ EDM $\cdot$ High speed wire EDM $\cdot$ HSWEDM $\cdot$ Material removal mechanism

\begin{tabular}{ll}
\multicolumn{2}{l}{ Abbreviations } \\
DC & Discharge crater \\
FP & Foot point \\
HSWEDM & High Speed Wire Electrical Discharge Machining \\
W & Wire \\
WE & Wire electrode \\
WP & Workpiece
\end{tabular}

List of Symbols

$d_{\mathrm{i}} \quad$ Diameters

$l_{\mathrm{i}} \quad$ Lengths

$n \quad$ Number of samples

$s \quad$ working gap

$t_{\mathrm{e}} \quad$ Discharge duration

$t_{\mathrm{i}} \quad$ Pulse duration

$t_{0} \quad$ Pulse interval time

$v_{\mathrm{w}} \quad$ Wire velocity

Kai Oßwald

kai.osswald@hs-pforzheim.de

1 Institute for Materials and Material Technologies, Pforzheim University, Tiefenbronner Str. 65, 75175 Pforzheim, Germany

2 National Instruments Germany GmbH, Ganghoferstraße 70b, 80339 Munich, Germany
$\Delta x \quad$ Relative movement of electrode during discharge duration $t_{\mathrm{e}}$

$\Delta x_{\mathrm{FP}} \quad$ Deformation of plasma channel

\section{Introduction}

High Speed Wire Electrical Discharge Machining (HSWEDM) has been described as a hybrid machining process in which different types of electric pulses lead to material removal [1]. In addition, a number of other features mark its difference to conventional wire EDM. The wire speed is typically set to $10-12 \mathrm{~m} / \mathrm{s}$ and the (molybdenum) wire is continuously wound back and forth between two spools. The high wire speed is improving flushing significantly. Consequently, the workpiece is usually not submerged in working medium. In order to enable the hybrid process to take place, specific working media were developed that typically have conductivity values around $3 \mathrm{mS} / \mathrm{cm}$. The pulse duration is commonly set to values between 20 and $50 \mu$ s and thus longer than in conventional wire EDM processes [2]. Recent publications on HSWEDM deal with aspects concerning the working medium $[3,4]$, phenomena in the working gap [5], or improvements of the machine design [6]. 
Due to the high velocity of the wire electrode, a significant relative movement of the plasma channel foot points on the electrodes occurs. This phenomenon has been observed before in the context of other EDM processes. Weingärtner et al. [7] analyzed discharge craters generated by wire electrical discharge dressing (WEDD) for different circumferential speeds in experiments and simulations. Traces of moving foot points were also observed in EDM-drilling by Munz [8] who determined that the craters are not only influenced by the relative motion and the pulse timing but also by the flushing intensity. Kunieda and Kameyama [9] have presented an exhaustive study on EDM material removal between moving electrodes. They investigated discharges and discharge craters for both working media, air and mineral oil. Their results show that the material removal on the anode as well as the cathode is influenced significantly by the relative motion. However, the effects are entirely different. To the best of the authors' knowledge, no research has been published investigating discharge craters or other material removal phenomena within the field of HSWEDM.

In adaption of the model of the EDM gap shown by Kunieda et al. [10], a model of the discharge situation was outlined which is depicted in Fig. 1. It resembles the "standard model" of Kunieda et al. [10] in many aspects. After the initiation of the discharge, the plasma channel evolves as well as a gas bubble surrounding it. The high current density at the foot points of the electrodes lead to melting of electrode material. The combination of these effects leads to material removal at the electrodes.

However, one fundamental difference to other electromachining processes - as mentioned above - consists in the high velocity of the wire electrode relative to the workpiece electrode. The absolute distance of this movement $\Delta x$ is dependent of the wire velocity $v_{\mathrm{w}}$ and the discharge duration $t_{\mathrm{e}}$ as shown in equation (1).
$\Delta x=v_{w} \cdot t_{e}$

For common values of $v_{\mathrm{w}}$ and $t_{\mathrm{e}}$ values of $\Delta x$ can easily reach several hundred micrometers. Consequently, the foot points of the plasma channel on at least one of the electrodes are expected to move multiples of their diameters during one discharge. Moreover, in most EDM processes, the workpiece electrode is switched as a cathode whilst in HSWEDM, it is typically switched as an anode which influences the occurring material removal mechanisms [11]. The material removal mechanisms are closely linked to the types of discharges that occur in a process. For the HSWEDM process, this has been investigated in [1]. The different pulse types found in that publication are shown in Fig. 2. In this research, the discharge craters and other material removal effects induced by these pulse types are investigated.

Discharge craters can be expected when dielectric (type 1) or thermal (type 2) breakdowns take place or when arcing occurs (type 3). All three pulse types generate a local plasma discharge (spark or arc) and high current density is a necessary characteristic in these cases. While dielectric breakdown (type 1) and arcing discharges (type 3 ) are common in conventional EDM processes, thermal breakdown discharges (type 2) are caused by an initial current flowing through the working medium and evolving to a discharge as described in more detail in [12]. Short circuit pulses (type 5) presumably can be observed when mechanical contact takes place or a current flow occurs for other reasons. In this case, discharge craters are not likely to be created. Due to the workpiece connection as an anode, a small part of the material removal takes place through anodic dissolution (type 4). Due to the comparably high conductivity of the working medium, small pulse currents can be observed even if the distance between the electrodes is several hundred micrometers. Therefore, no clear distinction between open circuit pulses and ECM pulses can be defined.
Fig. 1 Model of discharge situation in HSWEDM

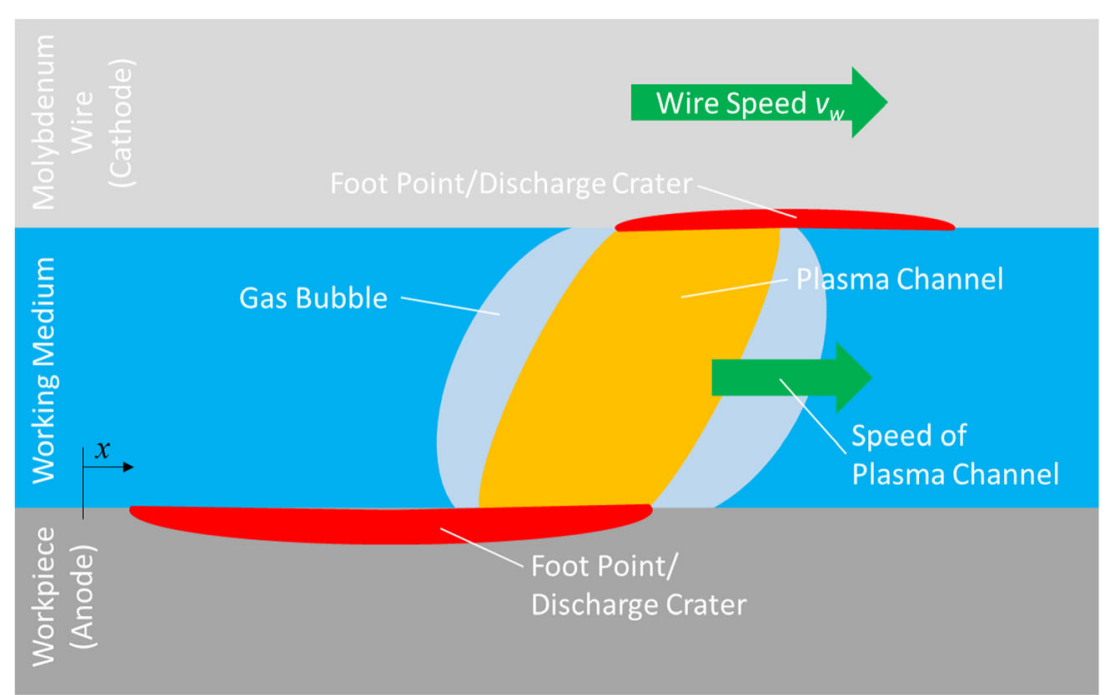


Fig. 2 Pulse types in HSWEDM according to [1]. Pulse types 1 through 3 can be regarded as discharges that are relevant for the generation of discharge craters

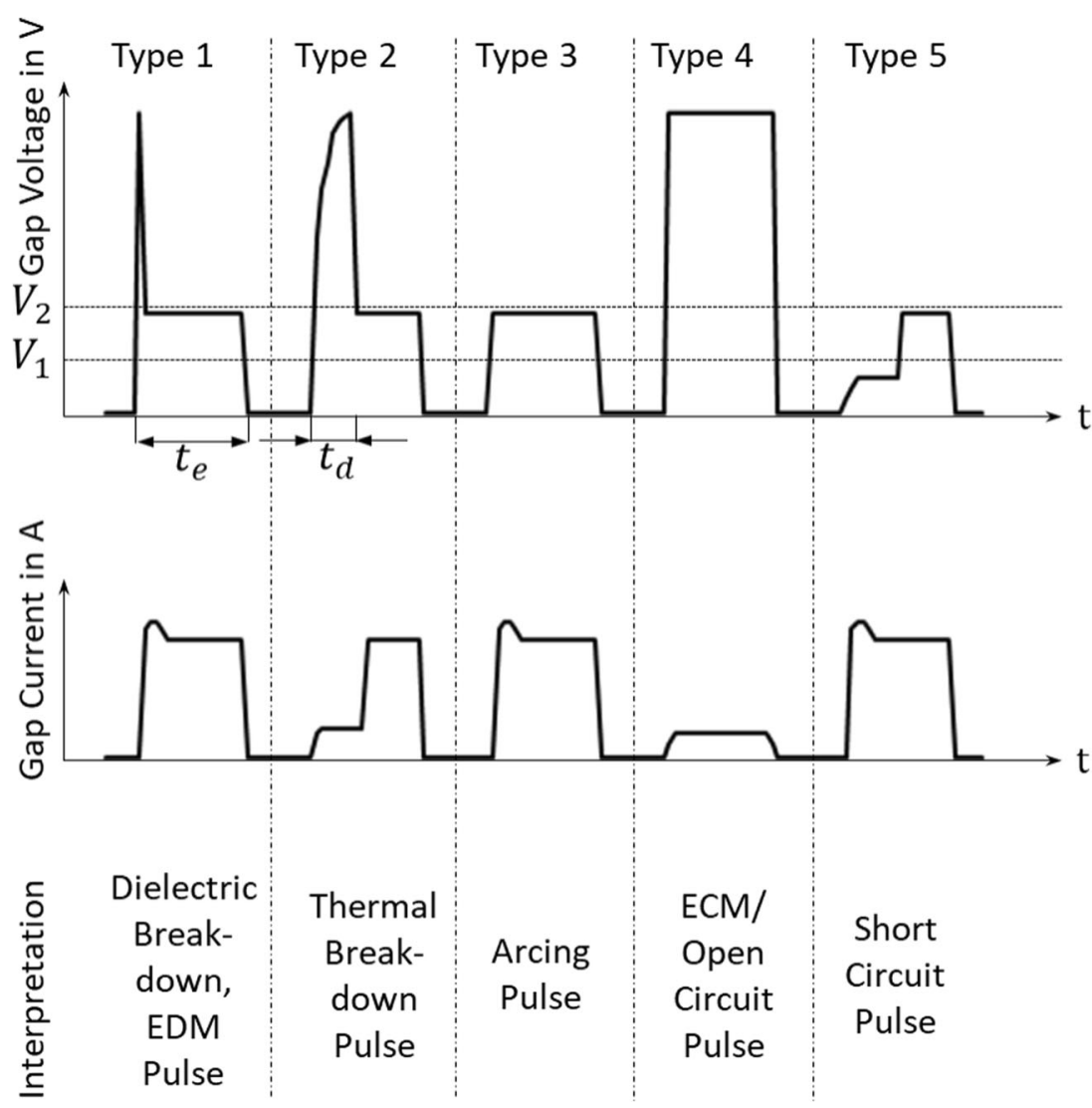

\section{Methods}

The experiments in this research were conducted on a standard HSWEDM machine (type DEM340) by KNUTH. The parameter set is listed in Table 1. A comparably small duty factor was chosen in order to increase the probability of single discharge craters on the samples' surfaces. The open circuit voltage $u_{\mathrm{i}}$ cannot be modified. The current parameter IP5 was measured to result in a discharge current of $i_{\mathrm{e}}=19 \mathrm{~A}$. The parameter "Feed Speed Adjust" that is influencing the process control is irrelevant in this context because the process was stopped after the first discharges on the probe's surface.

In order to observe the influence of the gas bubble, experiments were conducted with standard HWEDM working fluid as well as with air serving as a dielectric.

Table 1 Parameters of the HSWEDM process, used in this study

\begin{tabular}{llll}
\hline Parameter & Symbol & Value(s) & Unit \\
\hline Pulse duration & $t_{\mathrm{i}}$ & $30 ; 40$ & $\mu \mathrm{s}$ \\
Pulse interval time & $t_{0}$ & 200 & $\mu \mathrm{s}$ \\
Open circuit voltage & $u_{\mathrm{i}}$ & 70 & $\mathrm{~V}$ \\
Discharge current & $i_{\mathrm{e}}$ & 19 & $\mathrm{~A}$ \\
Wire velocity & $v_{\mathrm{w}}$ & 10 & $\mathrm{~m} / \mathrm{s}$ \\
Conductivity of working medium "wet" & $\sigma$ & 2.1 & $\mu \mathrm{S} / \mathrm{cm}$ \\
Working medium "dry" & & Air & \\
\hline
\end{tabular}

To obtain craters of single discharges, the moving wire was approached to a stainless steel (1.4571) sample workpiece with an electro-polished surface. In order to achieve a low feed speed perpendicular to the sample's surface, the wire was approached in an inclined angle. During the experiments, voltage and current signals were measured with an oscilloscope.

The surfaces of the samples were examined using a 3D profilometer and an optical microscope. The optical microscope (OLYMPUS BX51M) was equipped with a digital camera. A calibrated software was used to measure the lengths and widths of discharge craters. The profilometer (type MICROSPY PROFILE by FRT) allowed to investigate the three-dimensional shape of the discharge craters and to derive 2D profile sections using the proprietary MARK III software.

\section{Results and discussion}

\subsection{Craters of moving foot points due to discharges}

It was previously mentioned that the high relative velocity of the electrodes in HSWEDM has to lead to movements of the plasma channels' foot points on at least one of the electrodes. Consequently, craters of a more or less oval shape can be expected. However, it is very challenging to identify single 
discharge craters on the wire electrode (cathode). Consequently, the geometry of single discharge craters on the workpiece electrode (anode) was investigated in this research using an optical 3D profilometer. Experiments were conducted with two different discharge durations and two different working media ("dry": air; "wet" standard HSWEDM working medium with $\sigma=2.1 \mathrm{mS} / \mathrm{cm}$ ). The overall dimensions average length, average width, and average maximum depth of the craters ( $n \geq 15$ in all cases) are presented in Fig. 3. Despite the broad distributions of the values, some clear observations can be made. Increased pulse duration leads to an increased length of the discharge crater which confirms the dependency of the wire electrode movement. On a smaller scale, this also applies to the width of the craters which could be a result of the higher total pulse energy that was applied. Also, craters generated in air as a working medium tend to be longer (but not significantly wider) than craters generated in the liquid working medium. The maximum depth of the craters shows a considerable fluctuation in all cases, resulting in large standard distributions. The average maximum depth values are similar of all parameter sets except for $t_{\mathrm{i}}=30 \mu \mathrm{s}$ in air which is substantially smaller.

Comparing depth paths of individual single discharge craters is particularly challenging due to the large fluctuations in length and depth. Therefore, in order to allow a comparison, depth paths of the above-mentioned parameter sets were depicted as density scatter plots in Fig. 4. Herein, colors represent the relative occurrence of a certain depth along a certain lengthwise position for all measured depth paths. Thus, the lowest depicted values usually represent individual depth paths.

The "lengthwise crater position" on the abscissa indicates the distance from the crater edge in direction of the wire movement. In all cases, positive values of the crater depth can be

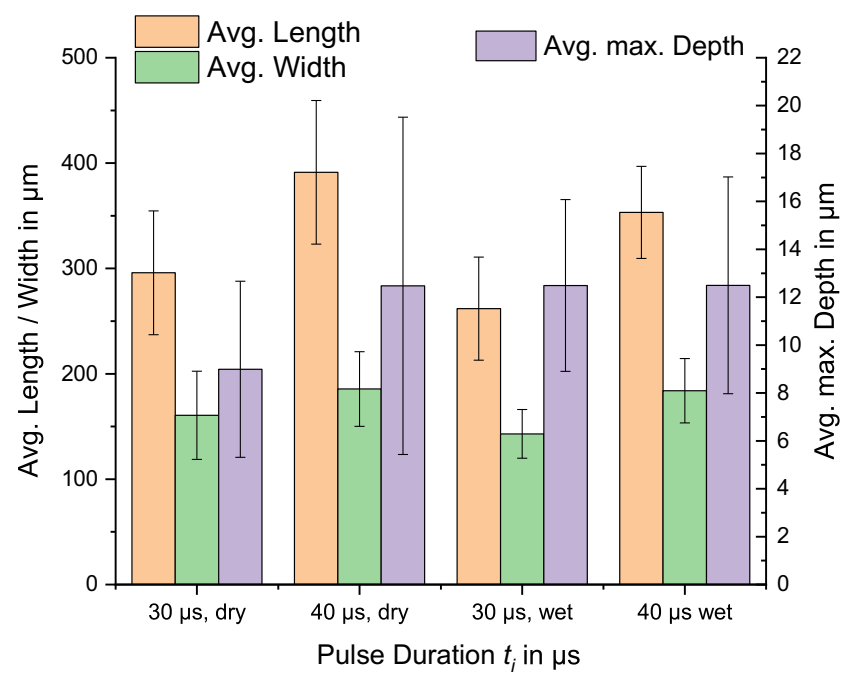

Fig. 3 Discharge crater dimensions on the workpiece electrode (anode) for different pulse durations and working media. Error bars indicate the standard distribution observed which indicates that resolidified molten material has exceeded the original level of the surface at these locations. Due to the differences in crater length, all diagrams tend to show higher density for smaller lengthwise positions. Diagrams (c) and (d) ("wet") indicate deeper craters than diagrams (a) and (b) which is not disclosed by the average values in Fig. 3 because they do not necessarily represent the absolute maximum of a crater. The deeper craters in diagrams (c) and (d) might be an effect of evaporating working medium.

The density plots in Fig. 4 can be interpreted as envelope curves. Within this range, individual craters can have significantly different depth paths. In the following, individual craters on the workpiece electrode will be presented that are representative for one parameter set, respectively. For each discharge crater, a 3D profile, a micrograph, and a longitudinal $2 \mathrm{D}$ profile (derived from the 3D measurement) will be shown.

In Fig. 5, craters of discharges through air ("dry") are presented. In all representations, the original workpiece surface can clearly be distinguished from the area affected by the discharge. For both pulse durations, the craters are surrounded of significant bulging. The depth path displays valleys and prominences including peaks of resolidified material almost reaching or exceeding the height of the original workpiece surface. As expected from the results presented above, the discharge crater (a) generated by the pulse duration of $t_{\mathrm{i}}=$ $30 \mu \mathrm{s}$ is considerably shorter $(l=346 \mu \mathrm{m})$ than the crater (b) generated by a pulse duration of $t_{\mathrm{i}}=40 \mu \mathrm{s}(l=454 \mu \mathrm{m})$.

The respective results for discharges through liquid working medium ("wet") are shown in Fig. 6. Here, the area surrounding the discharge crater in both cases shows traces of additional effects. The roughened surface lengthwise the craters is caused by anodic dissolution which will be presented later in this research piece. The shiny mark that is visible in the micrograph of crater (a) seems to be caused by mechanical contact of the two electrodes.

Both craters are surrounded by noticeably less bulging compared with their "dry" counterparts presented in Fig. 5. This is caused by evaporation of the liquid working medium. The expanding gas bubble leads to improved removal of the material [10]. As in the craters of Fig. 5, the depth paths show valleys and peaks, indicating a dynamic material removal and fast resolidification. Also, in this case, crater (a) $\left(t_{\mathrm{i}}=30 \mu \mathrm{s}\right)$ is shorter $(l=324 \mu \mathrm{m})$ than crater (b) with $l=385 \mu \mathrm{m}$, caused by the longer pulse duration of $t_{\mathrm{i}}=40 \mu \mathrm{s}$ of the latter. Both craters are shorter than their counterparts generated in air as a process medium which is an effect of the nonexistent bulging.

None of the discharge craters show an expected increase of the arc column diameter over time [10]. Probably, the plasma channel is already fully developed after a few microseconds. However, in all cases, the craters end with a narrowed tip (at the bottom of the micrographs) which seems to be caused by the collapsing plasma channel and/or gas bubble. 

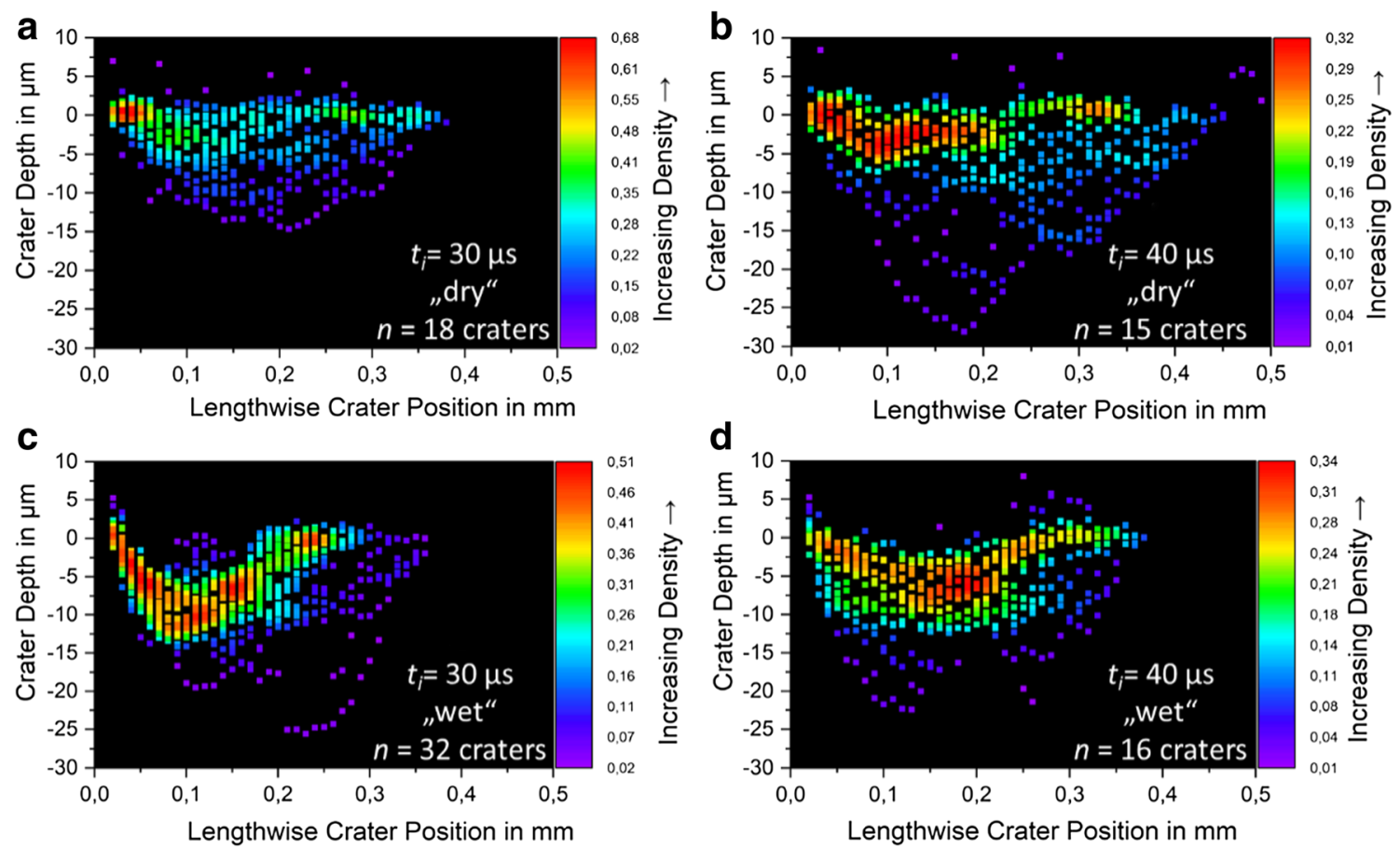

Fig. 4 Density scatter plots of the longitudinal depth paths of craters machined with different pulse durations and working media. Note that the color scale is different in every diagram

\subsection{Other material removal phenomena}

The hybrid nature of the HSWEDM process [1] entails different material removal mechanisms. Discharges with foot points on the workpiece certainly are contributing a major fraction of the total material removal. However, the experiments also revealed traces of other influences on the workpieces' surfaces. Figure 7 shows discharge craters with a nearly circular shape. Once again, these craters are only exemplary for a large variety of shapes occurring. Considering equation (1) suggests that in these cases, the foot point on the workpiece was static, and therefore, the foot point on the wire electrode was moving. Late breakdowns or interrupted discharges might be alternative explanations for this finding. Interruptions could have been caused by rapidly changing conditions in the gap. However, none of these phenomena were observed in the
3D-Profile

a)

$t_{i}=30 \mu \mathrm{s}$

Dry
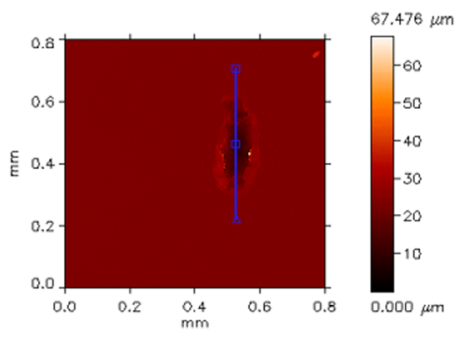

Micrograph
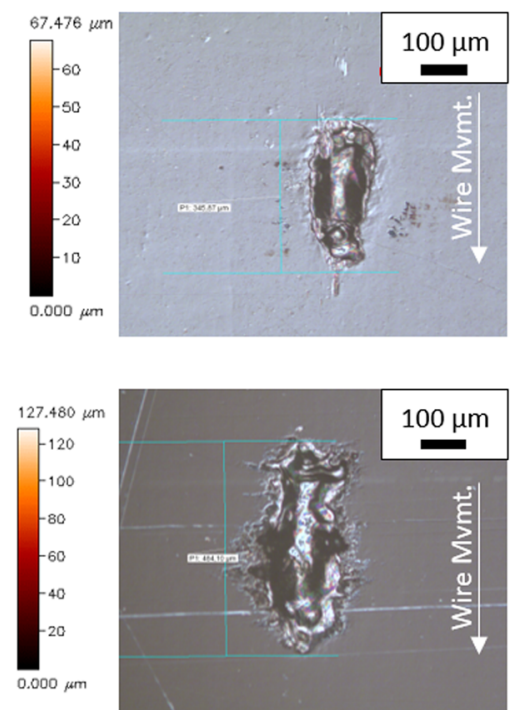

2D-Profile
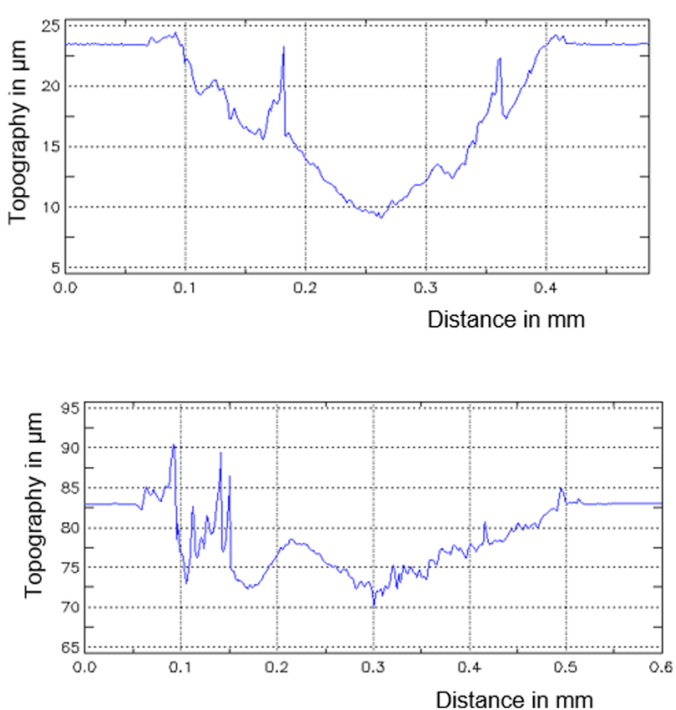

Fig. 5 Depictions of discharge craters on the workpiece electrode (anode) generated in the process medium air ("dry). Blue lines in the 3D profiles indicate the path of the $2 \mathrm{D}$ profile (starting from the top) 
3D-Profile

a) $t_{i}=30 \mu \mathrm{s}$ Wet

b) $t_{i}=40 \mu \mathrm{s}$ Wet

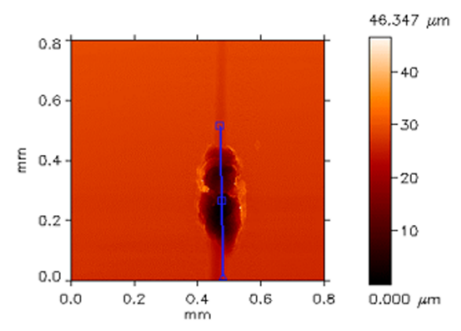

Micrograph
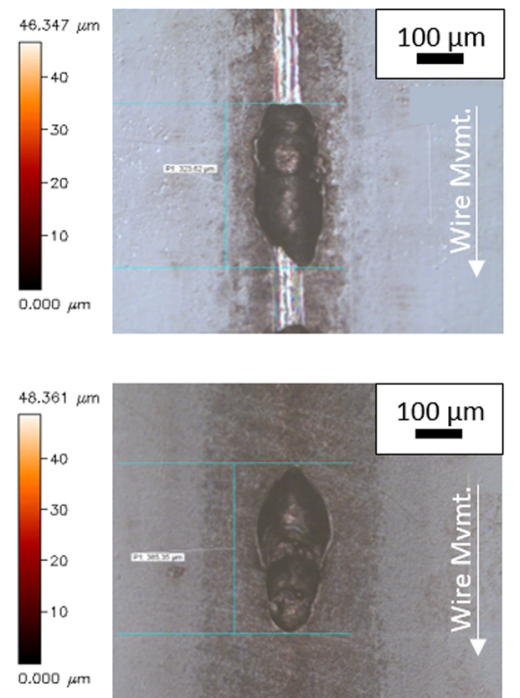

2D-Profile
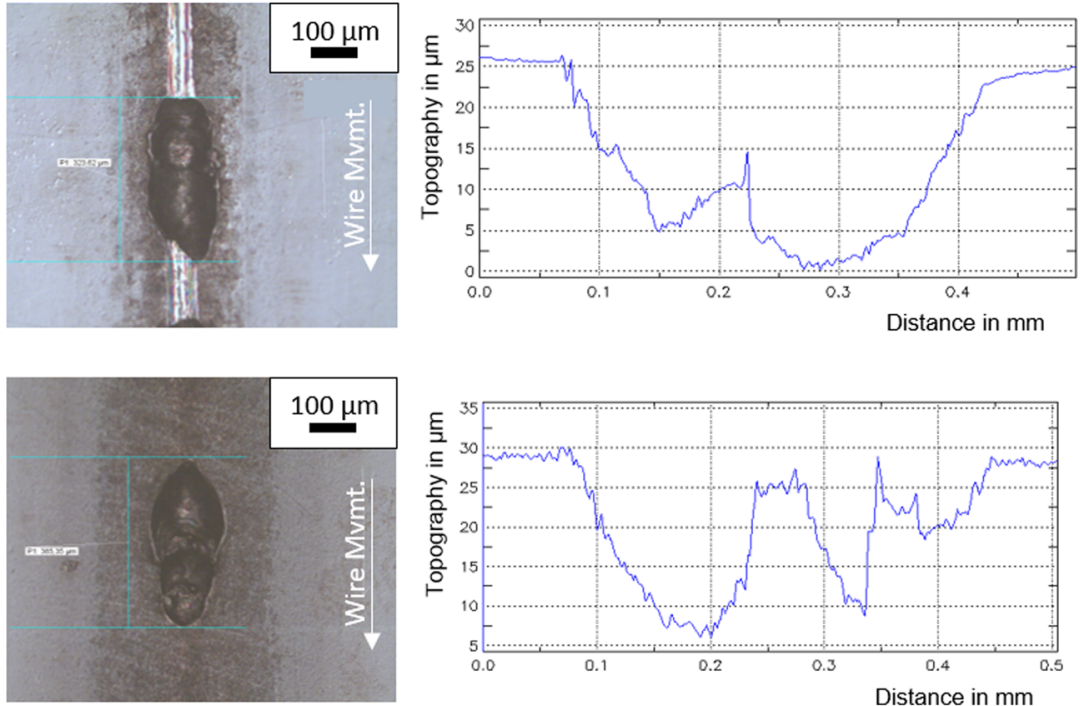

Fig. 6 Depictions of discharge craters on the workpiece electrode (anode) generated in the liquid process medium ("wet"). Blue lines in the 3D profiles indicate the path of the $2 \mathrm{D}$ profile (starting from the top)

current signals on the oscilloscope. The width of the craters is comparable with the craters presented in Chapter 3.1. with the identical pulse duration $t_{\mathrm{i}}=30 \mu \mathrm{s}$.

The occurrence of anodic dissolution was mentioned before in the description of Fig. 6. It usually does not appear in similar EDM processes due to the fact that for impulse durations $t_{\mathrm{i}} \geq 20 \mu \mathrm{s}$, the workpiece is usually connected as a cathode [13]. To investigate the influence of anodic dissolution in HSWEDM, the wire electrode was approached to the workpiece to a distance slightly larger than the HSWEDM working gap which had been determined prior to this experiment to be approximately $s \approx 25$
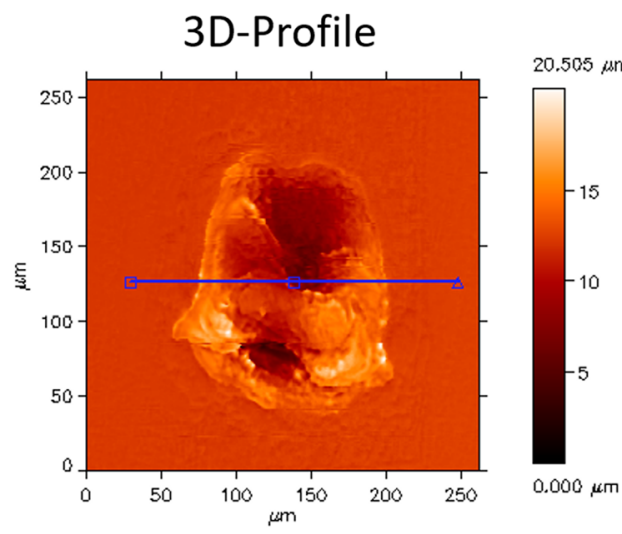

Micrograph

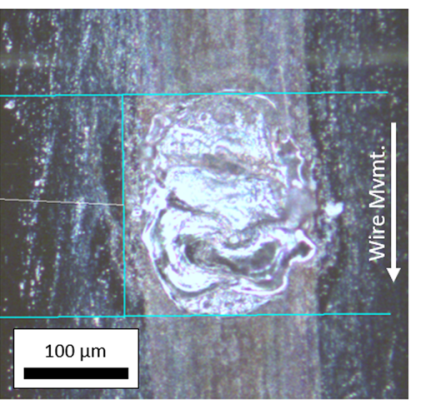

$52.088 \mu \mathrm{m}$

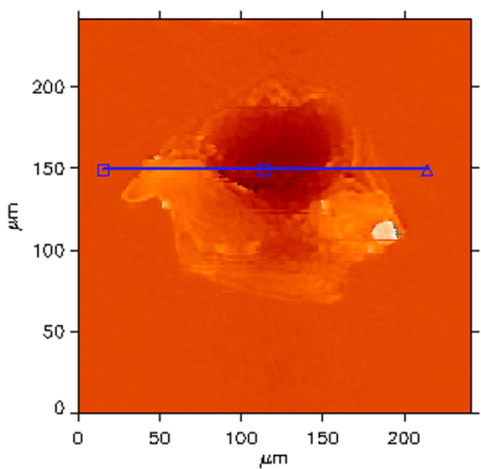

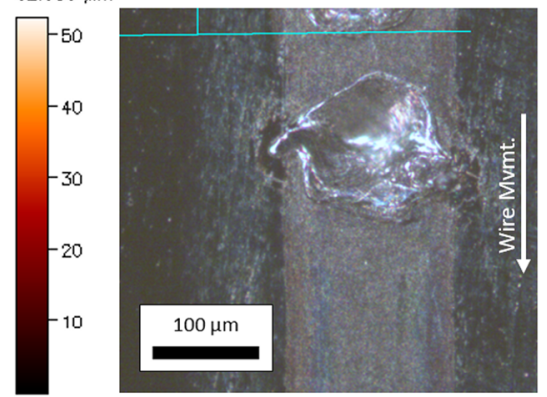

$0.000 \mu \mathrm{m}$
2D-Profile
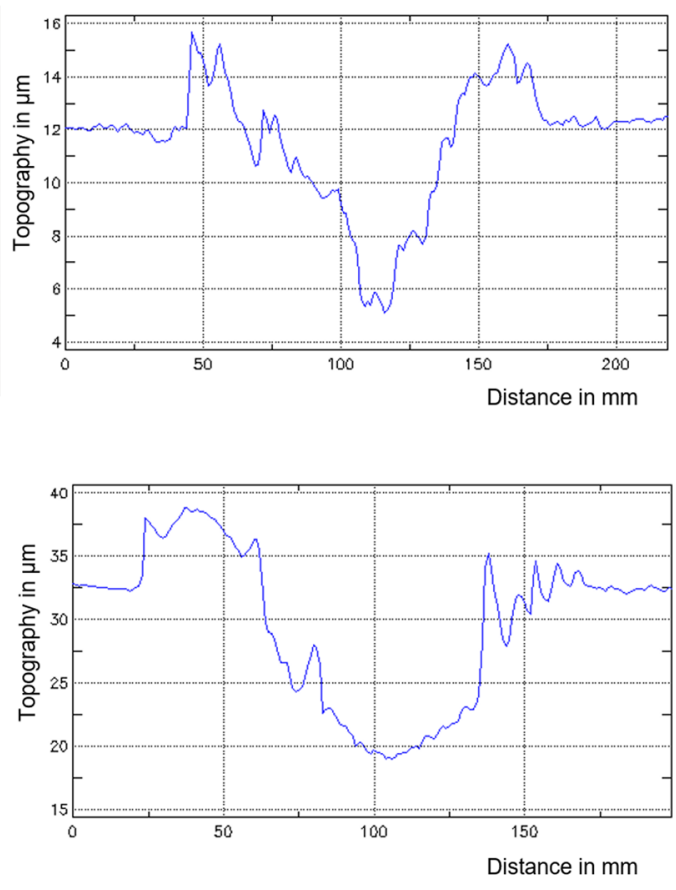

Fig. 7 Nearly circular discharge craters on the workpiece electrode (anode); $t_{\mathrm{i}}=30 \mu \mathrm{s}$; liquid working medium. Blue lines in the 3D profiles indicate the path of the $2 \mathrm{D}$ profile (starting from the left) 
3D-Profile

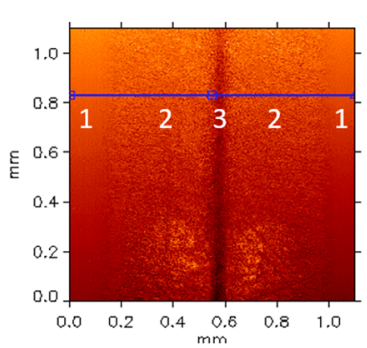

Micrograph

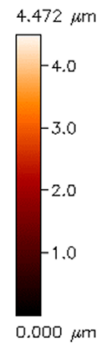

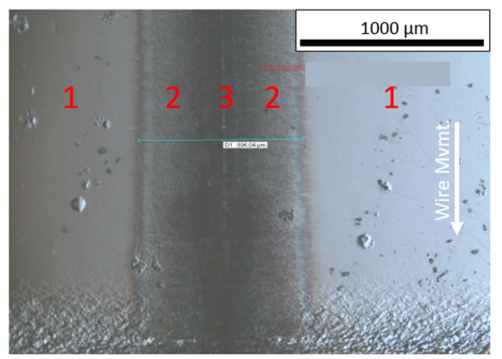

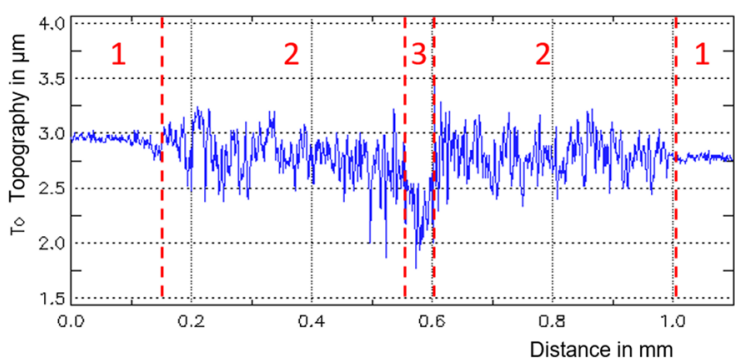

Fig. 8 Surface of workpiece electrode (anode) after purely electrochemical material removal $\left(t_{\mathrm{i}}=40 \mu \mathrm{s}\right)$. Blue line in the $3 \mathrm{D}$ profile indicates the path of the $2 \mathrm{D}$ profile

$\mu \mathrm{m}$. The process took place for $1 \mathrm{~s}$. Otherwise, all parameters were identical to the previous experiments. In Fig. 8, the workpiece surface after the experiment is shown. In all depictions, three areas can be distinguished. Area 1 represents the original workpiece surface; no surface modification took place in this region. In area 2, the electric field was sufficiently strong to start anodic dissolution. This leads to a roughening of the surface. Due to the round geometry of the wire, the electric field increases drastically towards the center. However, the roughness within area 2 is comparably uniform. In the center of the structure (area 3 , which is equivalent to the line of closest vicinity of wire and workpiece electrode) measurable- however littlematerial removal can be observed.

\subsection{Estimation of crater length on the wire electrode}

A description of the model presented in Fig. 1 that can be used for quantitative considerations is depicted in Fig. 9, including dimensions for the most important features.
For specific parameters, crater morphologies on cathode and anode can be fundamentally different; however, their sizes are in many cases similar [14]. Therefore, $d_{\mathrm{FP}, \mathrm{WE}}$ can be estimated to

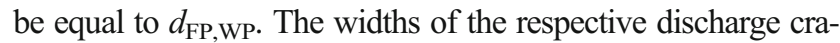
ters serve as approximate values for the plasma channel diameters $d_{\mathrm{FP}}$. Consequently, equation (1) can be complemented to

$v_{\mathrm{w}} \cdot t_{\mathrm{e}}=\Delta x=\left(l_{D C, W P}-d_{F P}\right)+\left(l_{D C, W E}-d_{F P}\right)$

Considering that the working gap was determined to be $s \approx$ $25 \mu \mathrm{m}$ and that all plasma channel diameters $d_{\mathrm{FP}}$ are well over hundred micrometers in diameter, it can be concluded that $\Delta x_{\mathrm{FP}}$ is negligible. Hence, the crater length on the wire electrode $l_{\mathrm{DC}, \mathrm{WE}}$ can be estimated by equation (2).

The graph in Fig. 10 shows a comparison between measured average values of the crater lengths on the workpiece $l_{\mathrm{DC}, \mathrm{WP}}$ (cf. Fig. 3) and calculated crater lengths on the wire electrode $l_{\mathrm{DC}, \mathrm{WE}}$. The lengths differ less than $10 \%$ when air was used as a working medium and less than $20 \%$ in liquid working medium. Thus, foot points in HSWEDM appear to move on both electrodes in a similar manner.
Fig. 9 Dimensions of discharge craters and foot points during a HSWEDM discharge

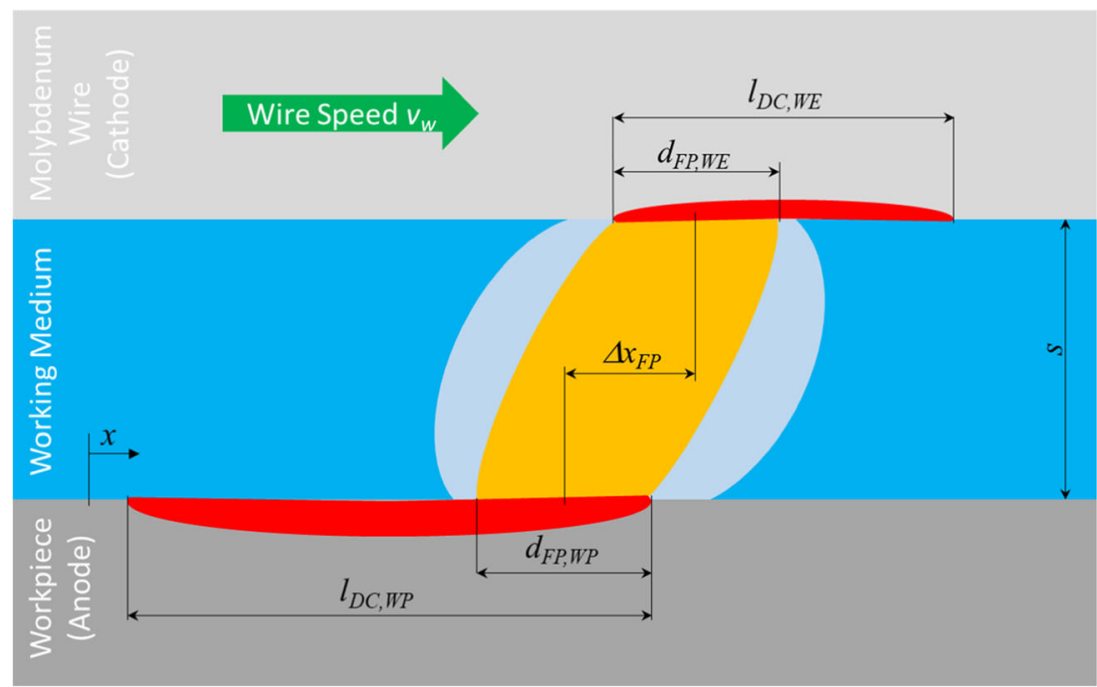




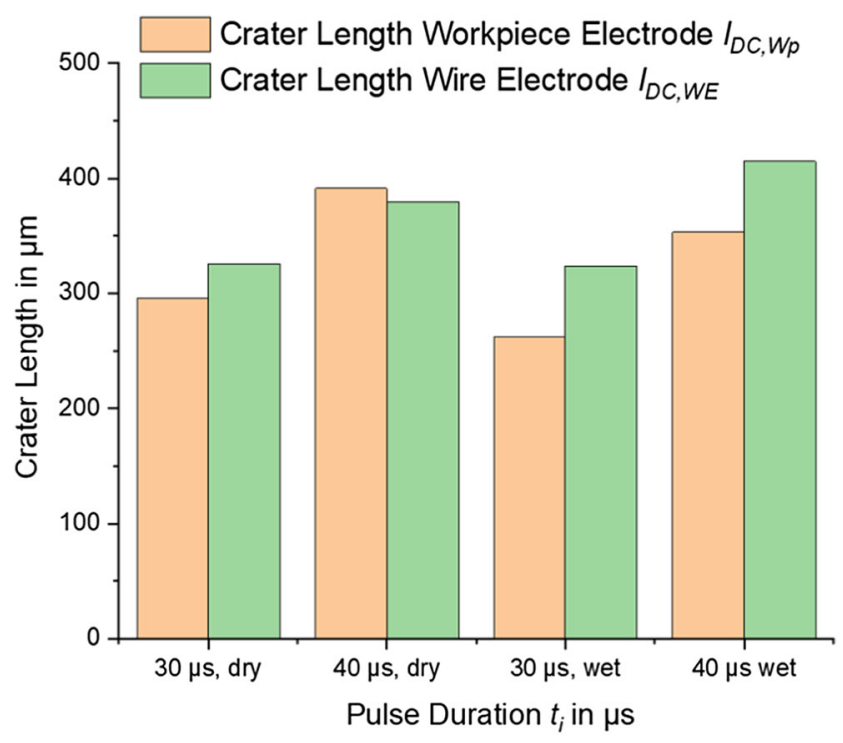

Fig. 10 Comparison between measured crater lengths on the workpiece electrode (average) and calculated crater lengths on the wire electrode

\section{Conclusions}

In this research, different removal mechanisms of High Speed Wire EDM were documented by their surface modifications on the workpiece electrode. Thus, the hybrid character of this manufacturing process was confirmed. Anodic dissolution and discharges that lead to circular craters appear to contribute only little to the overall material removal. Discharges with moving plasma channels causing elongated discharge craters are the predominant phenomenon that is induced by the high relative velocities between the electrodes. It can be assumed that this improves the efficiency of the material removal similar to the results presented by Weingärtner et al. [7]. However, this is to be shown in future research. By calculating estimated crater lengths on the wire electrode and comparing it with measured crater lengths on the workpiece electrode, it could be shown that the plasma channel moves similarly on both electrodes.

Acknowledgments The authors would like to thank Dr. Hans-Peter Schulze for providing the initial idea of this research.

Funding Open Access funding enabled and organized by Projekt DEAL.

Data availability Not applicable.

\section{Compliance with ethical standards}

Conflict of interest The authors declare that they have no conflict of interest.

Code availability Not applicable.

Open Access This article is licensed under a Creative Commons Attribution 4.0 International License, which permits use, sharing, adaptation, distribution and reproduction in any medium or format, as long as you give appropriate credit to the original author(s) and the source, provide a link to the Creative Commons licence, and indicate if changes were made. The images or other third party material in this article are included in the article's Creative Commons licence, unless indicated otherwise in a credit line to the material. If material is not included in the article's Creative Commons licence and your intended use is not permitted by statutory regulation or exceeds the permitted use, you will need to obtain permission directly from the copyright holder. To view a copy of this licence, visit http://creativecommons.org/licenses/by/4.0/.

\section{References}

1. Oßwald K, Lochmahr I, Schulze H-P, Kröning O (2018) Automated analysis of pulse types in high speed wire EDM. Procedia CIRP 68: 796-801. https://doi.org/10.1016/j.procir.2017.12.157

2. Oßwald K, Lochmahr I (2021) Effect of the relative velocity between electrodes in high speed wire EDM (HSWEDM). 20th CIRP Conference on Electro Physical and Chemical Machining (ISEM 2020). Procedia CIRP

3. Pan H, Liu Z, Li C, Zhang Y, Qiu M (2017) Enhanced debris expelling in high-speed wire electrical discharge machining. Int J Adv Manuf Technol 76:329-2920. https://doi.org/10.1007/s00170-017-0716-0

4. Wang W, Qiu M, Liu Z, Zhang M, Shao C (2020) Study on the influence of kerosene content on burn in high speed-wire cut electrical discharge machining dielectric fluid. Int J Adv Manuf Technol 51(10):599-3143. https://doi.org/10.1007/s00170-020-05187-z

5. Yueqin Z, Zhidong L, Lixia X, Wei W (2016) Interelectrode discharge mechanism in high-speed wire electrical discharge machining. Int J Adv Manuf Technol 84(9-12):2637-2647. https://doi.org/ 10.1007/s00170-015-7891-7

6. Ciwen H, Jinsheng Z, Jianyong L (2018) Ultra-long wire reciprocated-WEDM with dual tensile reels winded. Procedia CIRP 68:115-119. https://doi.org/10.1016/j.procir.2017.12.046

7. Weingärtner E, Wegener K, Kuster F (2013) Influence of workpiece circumferential speed in wire electrical discharge machining. Procedia CIRP 6:238-243. https://doi.org/10.1016/j.procir.2013. 03.063

8. Munz M (2015) Funkenerosives Bohren mit großen Aspektverhältnissen. $\mathrm{PhD}$ Thesis, in German language

9. Kunieda M, Kameyama A (2010) Study on decreasing tool wear in EDM due to arc spots sliding on electrodes. Precis Eng 34(3):546553. https://doi.org/10.1016/j.precisioneng.2010.01.009

10. Kunieda M, Lauwers B, Rajurkar KP, Schumacher BM (2005) Advancing EDM through fundamental insight into the process. CIRP Ann Manuf Technol 54(2):64-87. https://doi.org/10.1016/ S0007-8506(07)60020-1

11. Schulze H-P (2017) Importance of polarity change in the electrical discharge machining AIP Conference Proceedings, Bd 1896. AIP Publishing LLC., Melville, NY, S 50001

12. Oßwald K (2019) Effizientes Trennen mit High Speed Wire EDM. Galvanotechnik 117(7):1235-1240 in German language

13. Holsten M, Koshy P, Klink A, Schwedt A (2018) Anomalous influence of polarity in sink EDM of titanium alloys. CIRP Ann 67: 221-224. https://doi.org/10.1016/j.cirp.2018.04.069

14. Tamura T, Kobayashi Y (2004) Measurement of impulsive forces and crater formation in impulse discharge. J Mater Process Technol 149(1-3):212-216. https://doi.org/10.1016/j.jmatprotec.2003.10.063

Publisher's note Springer Nature remains neutral with regard to jurisdictional claims in published maps and institutional affiliations. 